\title{
GENERACIÓN DE OPCIONES DE DESTINO PARA SEMIPRODUCTOS DEL ACERO EN LAS EMPRESAS SIDERÚRGICAS
}

Doniel Jiménez Sánchez y José Arzola Ruiz

\section{RESUMEN}

Las mejores opciones de destino de hornadas de los semiproductos para la producción terminada de los talleres de la industria siderúrgica son aquellas en las que se minimiza el exceso de propiedades mecánicas con respecto a sus valores normados y se aseguran, por tanto, los valores requeridos de éstas. En este proceso se hace necesaria la estimación de las propiedades mecánicas de las hornadas a partir de su composición química y superficie transversal del producto terminado. En este trabajo la estimación se hizo mediante las redes neuronales de base radial regularizada, a partir de los datos acumulados de ensayos mecánicos en los talleres adoptados como caso de estudio. La utilización de estas redes permite disminuir los errores en la estimación de las propiedades mecánicas de los perfiles ligeros de acero. Se obtienen resultados satisfactorios en la generación de opciones de destino en un caso de estudio.

Palabras Claves: Dirección de la Producción, Selección de Materiales, Redes Neuronales de Base Radial, Regularización.

DOI: 10.23881/idupbo.021.1-8i 